\title{
Photoacoustic microscopy at super depths
}

\section{Lihong Wang}

Combining light and ultrasound in a single hybrid technology enables multiscale, high-resolution imaging deep into biological tissue.

With their ability to sense rich optical contrast in living tissue using harmless nonionizing radiation, commercial high-resolution 3D optical imaging techniques-such as confocal microscopy, two-photon microscopy, and optical coherence tomographyhave had a fundamental impact on biomedicine. These techniques, which are collectively known as ballistic imaging, depend on unscattered or minimally scattered photons. Its utility notwithstanding, ballistic imaging also has several limitations. In particular, diffraction constrains its resolution, and because living tissue scatters light, ballistic imaging techniques are unable to penetrate deeper than the so-called optical transport mean free path (around $1 \mathrm{~mm}$ in the skin).

For these reasons, developing novel ballistic imaging techniques with super-resolution imaging-i.e., at resolutions that surpass the diffraction limit-is important. Even more critical, however, is achieving super-depth high-resolution imaging, that is, beyond the transport mean free path. Furthermore, to understand the workings of biological systems spanning length scales from subcellular organelles to organs, biologists dream of multiscale imaging with a common signal source. One approach to deeply penetrating multiscale optical imaging in vivo is diffuse optical tomography, ${ }^{1}$ which takes advantage of multiply scattered photons to provide rapid functional imaging at super depths. But its spatial resolution is poor.

To overcome this problem, we have developed a technique called 3D tomographic photoacoustic microscopy (PAM). ${ }^{3,5,6}$ PAM involves optical irradiation, ultrasonic detection, and image formation. ${ }^{7-9}$ Tissue is irradiated by a short-pulsed laser, and the light absorbed by the material causes an increase in pressure via thermoelastic expansion. The increased pressure propagates through the tissue as an ultrasonic wave, known as a photoacoustic wave, which is detected by an ultrasonic transducer that produces electrical signals. The signals are then amplified,
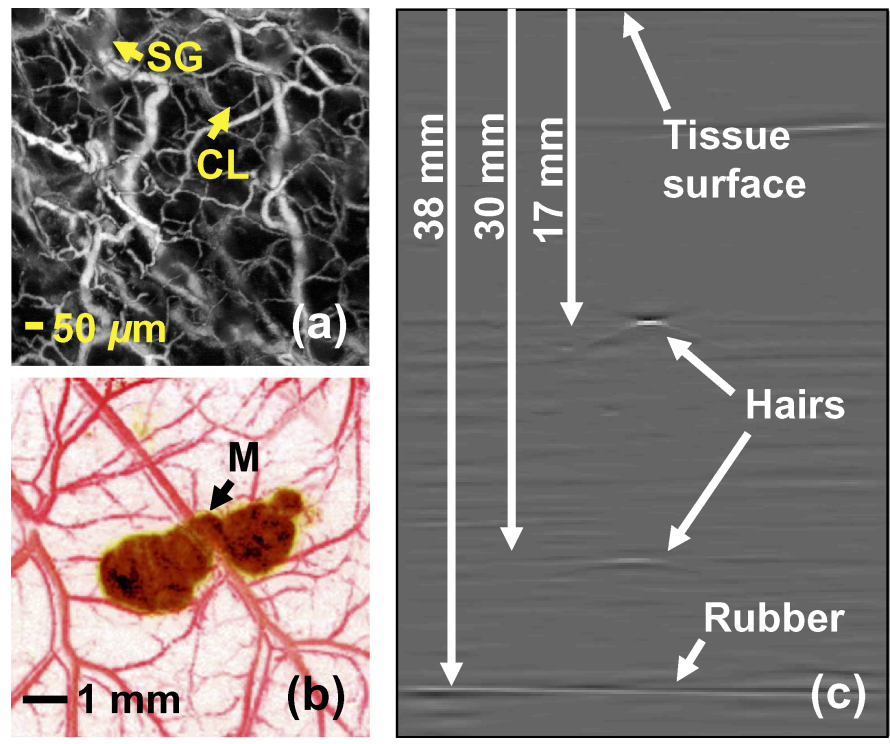

Figure 1. (a) In vivo image of blood capillaries acquired using an optical-resolution photoacoustic microscope. ${ }^{2}$ CL: Capillary. SG: Sebaceous gland. (b) Image of a melanoma and the surrounding blood vessels acquired using a $50 \mathrm{MHz}$ photoacoustic microscope. ${ }^{3}$ M: Melanoma. (c) Image acquired with a $5 \mathrm{MHz}$ photoacoustic microscope showing deep penetration in tissue. ${ }^{4}$

digitized, and transferred to a computer, where an image is formed.

Any absorbed photons-scattered or not-produce photoacoustic signals, and the ultrasonic scattering coefficient in tissue is 2-3 orders of magnitude less than its optical counterpart. As a result, PAM can provide deep penetration at high spatial resolution. In addition, the relative sensitivity of PAM to optical absorption is $100 \%$, much greater than that of other optical imaging technologies. ${ }^{7}$

Acoustic focusing in combination with time-resolved detection of photoacoustic waves ensures that PAM has 3D spatial resolution. The focal diameter and the bandwidth 
of the ultrasonic transducer determine the axial and lateral resolution, respectively. While each laser pulse produces a 1D depth-resolved image, scanning over the tissue produces multidimensional, speckle-free tomographic images.

PAM can simultaneously quantify concentrations of multiple chromophores, such as oxygenated and deoxygenated hemoglobin. This kind of quantification allows functional imaging of the concentration and oxygen saturation of hemoglobin. Both of these parameters are related to hallmarks of cancer such as angiogenesis ${ }^{10}$ and hypermetabolism. ${ }^{11}$ They can also be used to interrogate brain activity. ${ }^{12}$ In addition, extrinsic contrast can be used to provide molecular imaging of gene expression and biomarkers of disease. ${ }^{11,13}$

We have demonstrated the multiscale imaging capability of PAM in vivo with optical absorption contrast (see Figure 1). Our initial $50 \mathrm{MHz}$ photoacoustic microscope probes to a depth of $3 \mathrm{~mm}$ - filling the penetration void of ballistic imaging - at $15 \mu \mathrm{m}$ in axial resolution and $45 \mu \mathrm{m}$ in lateral resolution: see Figure $1(\mathrm{~b})$. We have also been able to scale the spatial resolution down to $5 \mu \mathrm{m}$ by focusing a light beam to less than the depth of one optical transport mean free path. This resolution allows in vivo imaging of capillaries, which are the smallest blood vessels: see Figure $1(\mathrm{a}) .^{2}$ The ultimate lateral resolution is optically diffraction limited, potentially yielding submicron resolution. We have also scaled the penetration limit up to centimeters, extending photoacoustic microscopy to macroscopy. For example, by employing a $5 \mathrm{MHz}$ ultrasonic transducer and an $804 \mathrm{~nm}$ light source, we were able to increase the penetration to $3 \mathrm{~cm}$ : see Figure $1(\mathrm{c}){ }^{4}$

In summary, PAM has transcended the fundamental depth limitation of conventional high-resolution optical imaging and is uniquely positioned to conduct multiscale imaging in vivo. As such, I believe that PAM will find broad application in both basic research and clinical care. Indeed, my co-workers and I are now working to bring photoacoustic imaging to the clinic. We recently received a grant from the National Institutes of Health to apply the technology to sentinel lymph node mapping in breast cancer. In addition, in collaboration with colleagues at Washington University, we plan to use the technique to probe melanoma, the deadliest form of skin cancer.

This work is sponsored in part by NIH grants R01 EB000712 and R01 NS46214 (Bioengineering Research Partnership).

\section{Author Information}

\section{Lihong Wang}

Optical Imaging Laboratory

Washington University in St. Louis

St. Louis, MO

Lihong Wang holds the Gene K. Beare Distinguished Professorship. He is a fellow of the American Institute for Medical and Biological Engineering, the IEEE, the Optical Society of America, and SPIE. He has published more than 157 journal articles and is the author of Biomedical Optics: Principles and Imaging. $\mathrm{He}$ has invented a range of imaging technologies, such as dark-field confocal photoacoustic microscopy.

\section{References}

1. B. Chance, R. R. Alfano, B. J. Tromberg, M. Tamura, and E. M. Sevick-Muraca, Optical tomography and spectroscopy of tissue VII, Proc. SPIE 6434, 2007.

2. K. Maslov, H. F. Zhang, S. Hu, and L. V. Wang, Optical-resolution photoacoustic microscopy for in vivo imaging of single capillaries, Opt. Lett. 33, pp. 929-931, 2008 doi:10.1364/OL.33.000929

3. H. F. Zhang, K. Maslov, G. Stoica, and L. V. Wang, Functional photoacoustic microscopy for high-resolution and noninvasive in vivo imaging, Nat. Biotechnol. 24 pp. 848-851, 2006. doi: $10.1038 /$ nbt1220

4. K. Song and L. V. Wang, Deep reflection-mode photoacoustic imaging of biological tissue, J. Biomed. Opt. 12, p. 060503, 2007. doi:10.1117/1.2818045

5. K. Maslov, G. Stoica, and L. V. Wang, In vivo dark-field reflection-mode photoacoustic microscopy, Opt. Lett. 30, pp. 625-627, 2005. doi:10.1364/OL.30.000625

6. H. F. Zhang, K. Maslov, and L. V. Wang, In vivo imaging of subcutaneous structures using functional photoacoustic microscopy, Nat. Protocols 2, pp. 797-804, 2007. doi: $10.1038 /$ nprot. 2007.108

7. L. V. Wang, Tutorial on photoacoustic microscopy and computed tomography, IEEE J. Sel. Top. Quant. 14, pp. 171-179, 2008. doi:10.1109/jstqe.2007.913398

8. M. H. Xu and L. V. Wang, Photoacoustic imaging in biomedicine, Rev. Sci. Instrum. 77, p. 041101, 2006. doi:10.1063/1.2195024

9. L. V. Wang and H. Wu, Biomedical Optics: Principles and Imaging, Wiley, Hoboken, NJ, 2007.

10. G. Ku, X. D. Wang, X. Y. Xie, G. Stoica, and L. V. Wang, Imaging of tumor angiogenesis in rat brains in vivo by photoacoustic tomography, Appl. Optics 44, pp. 770-775, 2005. doi:10.1364/AO.44.000770

11. M. L. Li, J. T. Oh, X. Y. Xie, G. Ku, W. Wang, C. Li, G. Lungu, G. Stoica, and L. V. Wang, Simultaneous molecular and hypoxia imaging of brain tumors in vivo using spectroscopic photoacoustic tomography, Proc. IEEE 96, pp. 481-489, 2008. doi:10.1109/jproc.2007.913515

12. X. D. Wang, Y. J. Pang, G. Ku, X. Y. Xie, G. Stoica, and L. V. Wang, Noninvasive laser-induced photoacoustic tomography for structural and functional in vivo imaging of the brain, Nat. Biotechnol. 21, pp. 803-806, 2003. doi:10.1038/nbt839

13. L. Li, R. J. Zemp, G. Lungu, G. Stoica, and L. V. Wang, Photoacoustic imaging of lacZ gene expression in vivo, J. Biomed. Opt. 12, p. 020504, 2007. doi: $10.1117 / 1.2717531$ 